\title{
A SURVEY OF THE RESULTS OF LACRIMAL STRICTUROTOMY*
}

\author{
BY \\ IVOR LLOYD
}

BRADFORD

ALTHOUGH the writer knows of no series of published cases illustrating the point, it is well recognised that the treatment by probing of epiphora due to disease of the lacrimal duct is extremely unsatisfactory. Ainsler has even said it may be easier to cure a detachment than a simple epiphora.

Stricturotomy for the relief of obstruction at the neck of the lacrimal sac is an established technique. in French clinics. "It is associated with the names of Poulard, Hartmann and Bastan.

English surgeons have not been interested to any great extent in the procedure and there do not appear to be publications of the results of a composite series of stricturotomy operations.

The writer, therefore, decided to give the method a trial on a reasonable number of cases, both from the point of view of verifying the results of the French ophthalmologists and because it presented interesting possibilities.

\section{Method}

The French method, used in the Hôpital Lariboisière, and explained with great clarity by Bastan, consists of opening the lower canaliculus with a Weber, Dugast, or similar knife, for $3 \mathrm{~mm}$., and then dilating the canaliculus with a number 3 sound; a knife with a bent handle is used where there is undue projection of the eyebrows. The stricturotomy knife was then passed down into the sac and engaged in the neck of the duct. It was then verticalized, after which the edge was rotated forwards through an angle of $45^{\circ}$ so that it faced somewhat forwards to prevent cutting the lower part of the canaliculus at its opening into the sac. Gum-elastic sounds from number 11 to 14 were then passed into the duct and the largest one left in situ for 10 minutes. Dilatation was continued at intervals of four to six weeks; the largest viable sound again being allowed to remain in situ for 10 minutes to stretch the opening.

The writer found that, with practice, the canaliculus could be dilated sufficiently to allow the passage of the knife with a slight or negligible slit. This was, in fact, preferable, as there was a considerable risk of occlusion of the punctum and/or the canaliculus from scarring if it was cut with a knife.

Dilatation was performed every two weeks for two months and then discontinued unless indicated by a return of symptoms.

* Received for publication, November 29, 1946. 


\section{Analysis of the Cases}

There were 31 patients which involved 33 stricturotomies. In order to give the method an extensive and searching trial a wide variety of types were included, the only exceptions being very old subjects or those in whom there was an excessive amount of purulent regurgitation. The ages of the patients were between 20 and 55 years. The duration of the symptoms averaged 2 years, although one or two patients had had epiphora for 10 years or more. One case was the result of extensive burns of the face which produced scarring of the lids and caruncle, but the canaliculi remained patent.

Mucoceles were present in four cases, and twelve of the patients had chronic low grade muco-purulent infection of the lacrimal passages. Ten of the patients had bilateral obstruction, and in two of them the bilateral operation was performed.

In all cases the condition of the nasal passages and sinuses was investigated.

Lipiodol X-ray photographs of the lacrimal passages were taken in some cases before and after the operation in order to determine the effects of the stricturotomy. It is regretted that this could not be done in all of the cases owing to the pressure of work and shortage of films in the war period.

\section{Results}

The French have claimed 100 per cent. cures in cases with no bony obstruction and 70 per cent. in those with bony obstruction. When pus or induration was present the results were correspondingly 3 to 7 per cent. less favourable.

Four of the writer's cases could not be traced later, so that the effective total of stricturotomies for analysis was 29.

Complete cure resulted in 15 of the patients (52 per cent.); "improvement" was obtained in 7 , i.e., patients considered the result of the treatment worth while. The percentage of symptomatic relief satisfactory to the patient was therefore 76 per cent.

There were seven failures. An analysis of these showed the following details :-

1. Impassable stricture, probably bony. This is unfortunately not diagnosable pre-operatively.

2. \& 3. Very tight strictures in which the stricturotome was passed with difficulty, dilatation being impossible later. Complete cure was obtained in both by the dacryocystorhinostomy operation of Toti.

4. One case was successful from a surgical point of view, and the sac and duct syringed through quite easily five months after the operation, but despite the absence of any other abnormality of the lacrimal passages the epiphora recurred three months later. 
5. In one subject infection was a feature with moderate mucopurulent regurgitation. The operation of stricturotomy and subsequent dilatations kept the epiphora and infection in check, but they recurred at the termination of the treatment.

6. The patient with the scars due to burns was shown to have a medium sized mucocele on radiological investigation, but stricturotomy was unsuccessful as a lacrimal abscess developed shortly after the treatment had commenced, and the method had to be abandoned.

7. As a matter of interest and to produce visual evidence of the presence of a stricture, the writer performed an open stricturotomy on one subject. Continuous dilatation was maintained for a week after the operation by a bougie, by which it was hoped to avoid periodic dilatation later. Although the minimum of trauma was inflicted and the sac was closed with very fine cat-gut sutures through the superficial layers only, the subsequent scarring at the lower end of the canaliculus and in the sac led to a return of the epiphora some months afterwards.

\section{Discussion}

The operation of stricturotomy has many advantages over the usual methods of treatment of lacrimal obstruction.

It can be performed in the out-patient clinic, thus avoiding the necessity for admission to the wards of a hospital. It is a relatively minor operation leaving no residual unsightly scarring of the face. If the method fails it does not prejudice the exhibition of any other surgical procedure on the lacrimal passages. Finally, it is of especial value in cases where the obstruction is of a fibrous type in which ordinary probing so often proves unsuccessful.

The writer, in agreement with the French clinicians, maintains that the results are as good, if not better, than those obtained with other methods.

On the other hand, the operator must be prepared to devote at least one half day a week to the "lacrimal clinic" if many cases are to be treated, and the large number of dilatations involved renders the method rather tedious. In addition, despite the use of local anaesthesia, dilatation was a slightly painful procedure. It was considered inadvisable to inject an anaesthetic on each occasion owing to the soreness of the skin produced.

Considering the above objections the writer was pleasantly surprised to find that the patients were anxious to continue the treatment, indicating their appreciation of the relief of symptoms.

The presence of a stricture at the junction of the lacrimal sac and the naso-lacrimal duct has been denied, but the writer has shown by radiological findings and also at the open operation that such an abnormality exists in many cases of epiphora. After passing a 
stricturotome it was quite evident that some constriction had been relieved because a large probe or bougie, previously impassable, then entered the duct without undue force being required to make it do so.

\section{Summary}

The results of 33 stricturotomy operations are analysed, and the causes of failure detailed.

Modifications of the French technique are suggested.

Its advantages compared with other methods of treating lacrimal obstruction are discussed.

The writer wishes to express his grateful appreciation to Mr. John Foster of Leeds, for introduction to the method and the reference quoted, and correspondence on the subject, also for permission to publish these cases treated in his clinic.

\section{REFERENCE}

Bastan, N.-Ann. d'Ocul., 171, Part 1, January, 1934.

\section{ANNOTATIONS}

\section{Areopagitical}

"Areopagitica : a speech for the liberty of unlicensed printing" was published by Milton in 1664 as an answer to an order in Parliament that " no book, pamphlet, or paper should be henceforth printed, unless the same was first approved and licensed by such, or at least by one of such, as shall be thereto appointed.

"Give me," said Milton, "the liberty to know, to utter, and to argue freely according to conscience, above all other liberties.

This was the beginning of a campaign for freedom of the press which ended in 1695, when William the Third ratified the bill for ending censorship. Everyone who writes or publishes owes a debt to Milton. Had we been editing the British Journal of Ophthalmology three hundred years ago we tremble to think of what might have happened to us after the publication of any particular number. Doubtless we should have been acquainted with the pillory, our nose might have been slit, our ears cut off, we might have been flogged at the cart tail from our domicile or consulting room to the printers' offices every other month; and the fines demanded of us would have soon landed us in the Fleet, or debtor's prison. But, happily for us, these barbarities are a thing of the past. And though 